\title{
Tanshinone IIA prevents platelet activation and down-regulates CD36 and MKK4/JNK2 signaling pathway
}

\author{
Hua Wang ${ }^{1,2}$, Lin Zhong ${ }^{2}$, Shaohua Mi ${ }^{2}$, Nianpeng Song ${ }^{1,2}$, Wei Zhang ${ }^{1}$ and Ming Zhong ${ }^{1 *}$ (D)
}

\begin{abstract}
Background: Tanshinone IIA (TS IIA), a multi-pharmaceutical compound from traditional Chinese herb, is effective for treatment of atherothrombosis. However, the underlying mechanisms of TS IIA-mediated anti-platelet activation effect are still poorly understood. As shown in our previous study, platelet-derived microvesicles (PMVs) generated in response to oxidant insult could activate CD36/mitogen-activated protein kinase kinase 4/Jun N-terminal kinase 2 (CD36/MKK4/JNK2) signals and lead to platelet activation. The present study aims to investigate the effect of TS IIA on platelet activation and the possible mechanisms.

Methods: The production of PMVs induced by Interleukin 6 (IL-6) was detected by flow cytometry. We performed activating studies of platelets with PMVs derived from IL-6-treated platelets (IL-6-PMVs) in vitro. Sometimes, platelet suspensions were incubated with serial concentrations of TS IIA for 15 min before being stimulated with IL-6-PMVs. Expression of platelet integrin $a_{\| 1} \beta_{3}$ and CD36 was detected by flow cytometry. Phosphorylation of MKK4 and JNK were detected by immunoblotting.
\end{abstract}

Results: Here we demonstrated firstly that TS IIA could prevent platelet activation induced by PMVs and downregulates CD36 and MKK4/JNK2 signaling pathway. CD36 may be the target of atherosclerosis (AS)-related thrombosis.

Conclusions: This study showed the possible mechanisms of TS IIA-mediated anti-platelet activation and may provide a new strategy for the treatment of AS-related thrombosis by targeting platelet CD36.

Keywords: Tanshinone IIA, Platelet activaton, CD36, Atherothrombosis

\section{Background}

Atherothrombosis is the leading cause of morbidity and mortality worldwide. Tanshinone IIA (TS IIA), a pharmacological active components extracting from the rhizome of the Chinese herb Salvia miltiorrhiza Bunge, has been used widely for effective treatment of atherothrombosis in traditional Chinese medicine for a long history $[1,2]$. However, its underlying molecular mechanisms of anti-thrombotic effect are still poorly understood. Platelet activation has been

\footnotetext{
*Correspondence: zhongmingsdu2018@163.com.cn

'The Key Laboratory of Cardiovascular Remodeling and Function Research, Chinese Ministry of Education, Chinese National Health Commission and Chinese Academy of Medical Sciences, The State and Shandong Province Joint Key Laboratory of Translational Cardiovascular Medicine, Department of Cardiology, Qilu Hospital of Shandong University, No 107 Wenhua West Road, Ji'nan 250012, China

Full list of author information is available at the end of the article
}

implicated as central components of the Virchow's triad for thrombus formation [3]. Platelet-derived microvesicles (PMVs) have a critical role in activation of platelets [4]. It is well established that PMVs, carrying inflammation factors, oxidation factors, various growth factors and coagulation factors, can participate in a wide range of pathophysiological process $[5,6]$. Similarly, TS IIA has been reported to have multi-pharmaceutical activities, such as antiinflammatory capacity [7], antioxidant properties [8] and the ability to prevent platelet activation [9]. Moreover, Tang et al. [10] had showed that TS IIA attenuates atherosclerosis in ApoE (-/-) mice through down-regulation of CD36 expression. CD36, a class B scavenger receptor, promotes platelet activation by binding with PMVs. As shown in our previous study, PMV-CD36 complex could activate mitogen-activated protein kinase kinase 4/Jun $\mathrm{N}$-terminal 
kinase 2 (MKK4/JNK2) signals and lead to platelet activation [11]. We hypothesized that TS IIA could inhibit platelet activation by suppressing the effects of MKK4/JNK2 signaling pathway mediated by PMV-CD36 complex, which has never been reported.

The present study intends to investigate whether TS IIA could prevent platelet activation induced by PMVcomplex and down-regulates MKK4/JNK2 signaling pathway. PMV-CD36 complex could be the target of atherosclerosis (AS)-related thrombosis.

\section{Methods}

\section{Reagents}

TS IIA was obtained commercially (Xi'an Honson Biotechnology, China). IL-6 (0203B16) was from PEPROTECH, USAInhibitory reagents. SP600125 was from Calbiochem (San Diego, CA, USA).

\section{Reagents used for flow cytometry}

Phycoerythrin (PE)- cy ${ }^{\text {Tm}} 5$-conjugated mouse anti-CD41a antibody (clone HIP8), fluorescein isothiocyanate (FITC)conjugated PAC-1, PE-conjugated anti-CD36 (clone CB38) and PE-conjugated CD36 isotype control (clone G155-228) were purchased from BD Biosciences/Pharmingen (San Jose, CA, USA).

\section{Reagents used for western-blot analyses}

Monoclonal rabbit anti-MKK4, phospho-MKK4 (pMKK4), c-Jun NH2-terminal kinase (JNK) and mouse anti-phospho-JNK (p-JNK) were from Cell Signaling Technology (Danvers, MA, USA).

\section{Platelet isolation}

Blood from healthy volunteers was collected into sodium citrate anticoagulant. Donors had not taken aspirin or NSAIDs for 2 weeks [11, 12]. At room temperature,
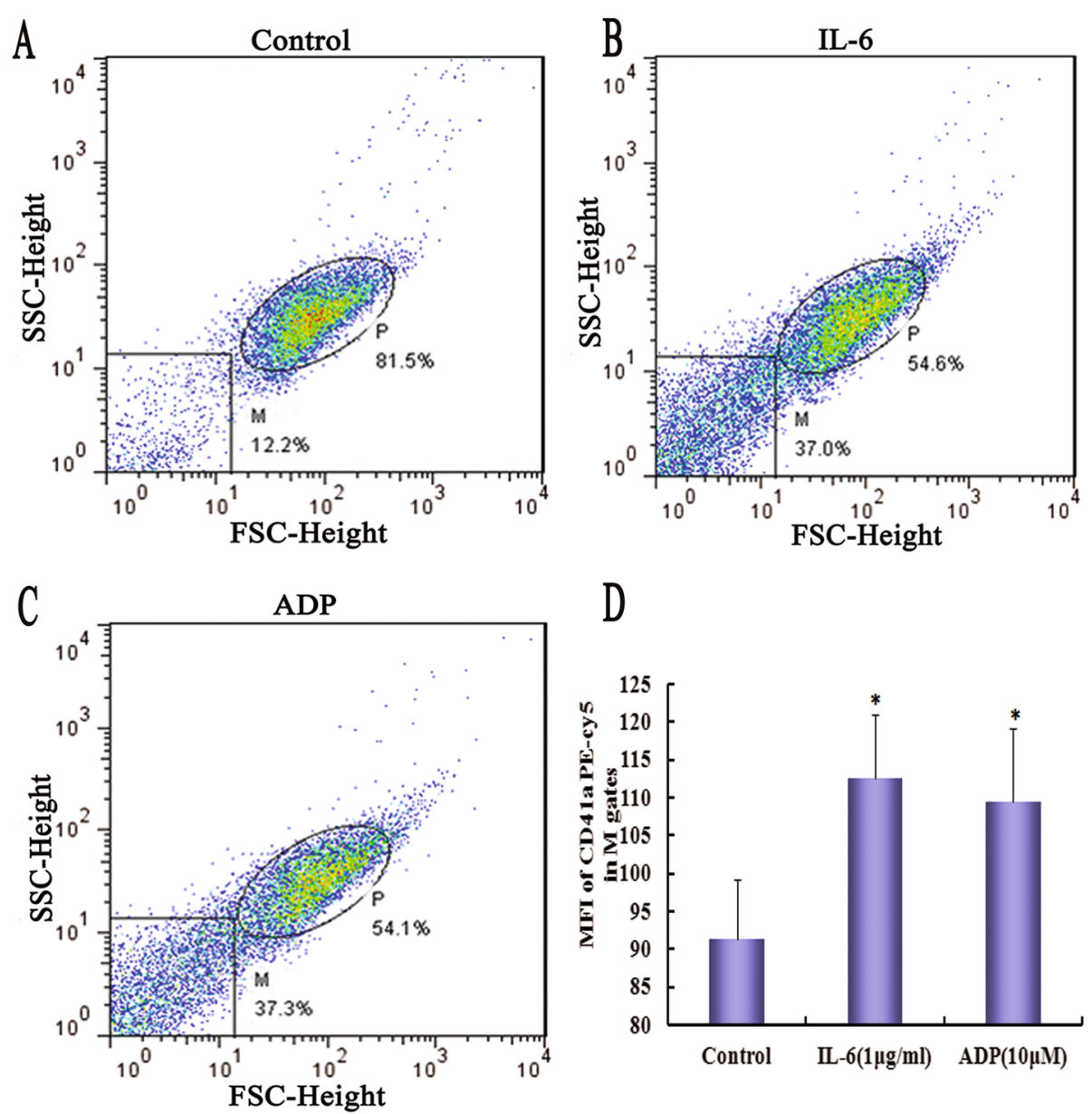

Fig. 1 Flow cytometric analysis of platelet-derived microvesicles (PMVs). Resting platelet suspensions $\left(1 \times 10^{6} / \mathrm{mL}\right)$ were treated with buffer control a, $1 \mu \mathrm{g} / \mathrm{mL}$ Interleukin 6 (IL-6) b or $10 \mu \mathrm{mol} / \mathrm{L}$ adenosine diphosphate (ADP) c for $60 \mathrm{~min}$ at $37^{\circ} \mathrm{C}$. Representative flow cytometric scatter plot (M in Fig. 1a-c). P and M indicate the gates for platelets and PMVs, respectively. $\mathbf{d}$ Mean fluorescent intensity (MFI) of CD41a PEcy5 in M gates. Data are means \pm SD from 3 separate experiments. ${ }^{*} P<0.05$ compared with control 
platelet-rich plasma (PRP) was separated by centrifugation $(10 \mathrm{~min}$ at $120 \mathrm{~g})$. Platelets were obtained from PRP after centrifugation and washed 3-times with equal volumes of modified Tyrode's Buffer, composed of $137 \mathrm{mmol} / \mathrm{L} \mathrm{NaCl}$, $2.7 \mathrm{mmol} / \mathrm{L} \quad \mathrm{KCl}, \quad 12 \mathrm{mmol} / \mathrm{L} \quad \mathrm{NaHCO} 3, \quad 0.4 \mathrm{mmol} / \mathrm{L}$ $\mathrm{NaH} 2 \mathrm{PO} 4,5 \mathrm{mmol} / \mathrm{L}$ HEPES, $0.1 \%$ glucose and $0.35 \%$ bovin serum albumin (BSA), $100 \mathrm{nmol} / \mathrm{L}$ PG-E1 (SigmaAldrich), $\mathrm{pH}$ 7.2. Platelet suspension was adjusted to $1 \times$ $10^{6} / \mathrm{mL}$. The final platelet was resuspended at a concentration of $1 \times 10^{6}$ platelets $/ \mathrm{mL}$ in modified Tyrode's buffer.

\section{Isolation of PMVs derived from platelet supernatant} Platelet suspension $\left(1 \times 10^{6} / \mathrm{mL}\right)$ was exposed to buffer control, $1 \mu \mathrm{g} / \mathrm{mL}$ IL-6 (Pepro Tech, USA) or $10 \mathrm{~mol} / \mathrm{L}$ adenosine diphosphate (ADP) for $60 \mathrm{~min}$ at $37^{\circ} \mathrm{C}$. Culture supernatants were collected, and nonviable cells and cell fragments were removed by centrifugation at $3000 \mathrm{~g}$ for $10 \mathrm{~min}$. Subsequently, the supernatants were then sedimented at $15,000 \mathrm{~g}$ for $60 \mathrm{~min}$ at $4{ }^{\circ} \mathrm{C}$ to pellet PMVs. Then the PMV pellets were washed twice to avoid contamination of IL- 6 and resuspended in modified Tyrode buffer. The PMVs were characterized by flow cytometry (BD Biosciences) $[11,13]$ and the protein content was measured by the Bradford Protein Assay Kit (Beyotime, Jiangsu, China).

\section{Flow cytometric measurement of platelet integrin $\alpha_{11 b} \beta_{3}$ (PAC-1) and CD36 expression}

Washed platelet suspensions $\left(1 \times 10^{6} / \mathrm{mL}\right)$ were incubated with IL-6-PMVs (MVs derived from IL-6 stimulated platelets). In some studies, to elucidate the effect of TS IIA on platelet activation or platelet CD36 expression, resting platelets were treated independently with serial concentrations of TS IIA for 15 min before exposure to PMVs. Expression of platelet integrin $\alpha_{\mathrm{IIb}} \beta_{3}$ and CD36 was analyzed by flow cytometry. Activated platelet integrin $\alpha_{\mathrm{IIb}} \beta_{3}$ were labeled by adding $5 \mu \mathrm{L}$ PEcy5-conjugated anti-CD41a antibody and $5 \mu \mathrm{L}$ FITC-conjugated PAC-1 antibody. $5 \mu \mathrm{L}$ PEcy5-conjugated anti-CD41a antibody and $5 \mu \mathrm{L}$ PEconjugated anti-CD36 antibody were used to detect platelet CD36 expression in the dark for $15 \mathrm{~min}$. MKK4/JNK2 mediated signaling pathways were blocked using JNK inhibitor SP600125.

\section{Western blot analysis}

Protein sample preparation and western blot analysis were performed as described in previous studies [11] with primary antibodies against p-MKK4 or total MKK4 and p-JNK or total JNK (Cell Signaling Technology, Danvers, MA), followed by anti-IgG horseradish peroxidase-conjugated secondary antibody.

\section{Statistical analysis}

Statistical analyses were carried out using SPSS 18.0 (SPSS Inc., Chicago, IL). Data are presented as means \pm SD. Comparison among groups involved an ANOVA with post-hoc least-significant differences $t$ test (for continuous data). A $P$ value less than 0.05 was considered statistically significant.

\section{Results}

IL-6 stimulation increases the formation of PMVs

Flow cytometric analysis of washed platelets showed an increase in the formation of PMVs after treatment with $1 \mu \mathrm{g} / \mathrm{mL}$ IL-6 or $10 \mu \mathrm{mol} / \mathrm{L}$ traditional agonist ADP (Fig. 1). The flow cytometric scatter patterns indicate the formation of PMVs in the lower lefthand gate of the scatter plot ( $\mathrm{M}$ gates in Fig. 1a-c). The PMVs were identified by labeling with PEcy5-conjugated antiCD41a antibody. Compared with untreated platelets, the mean fluorescence intensity (MFI) of CD41a in M gates increased significantly in the IL- 6 or ADP treated platelets (Fig. 1d).

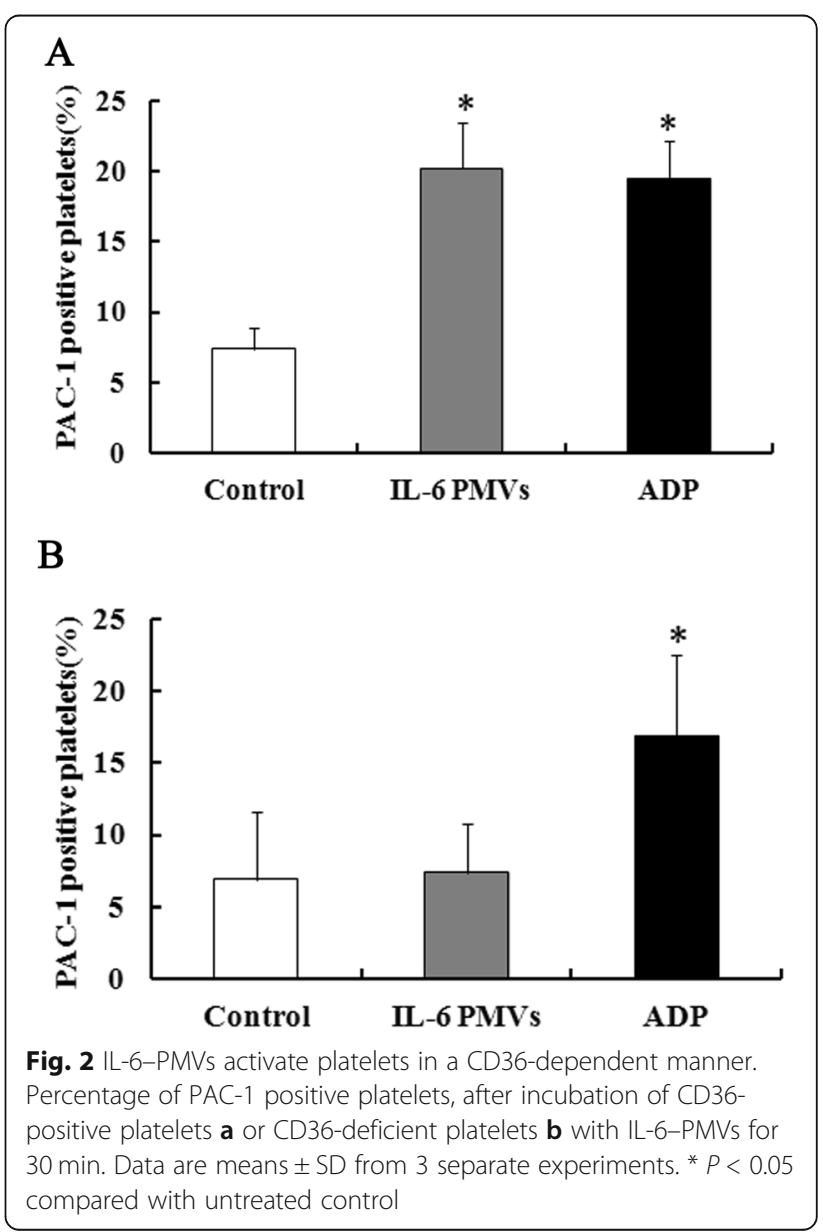




\section{IL-6-PMVs induced platelet activation in a CD36- dependent way}

It is reported that $3-4 \%$ of Asian are platelet CD36deficient subjects [14]. We found 2 CD36-deficient volunteers. The CD36-deficient platelets, which were identified by flow cytometry, unable to bind PE-conjugated anti-CD36 antibody [11]. We incubated CD36-positive and CD36-deficient platelets with IL-6-PMVs $(30 \mu \mathrm{g} /$ $\mathrm{mL}$ ) for $30 \mathrm{~min}$ respectively. Platelet activation is recognized as a conformation change in integrin $\alpha \operatorname{IIb} \beta 3$. The percentage of PAC-1 (recognizing the activated platelet integrin $\alpha \mathrm{IIb} \beta 3$ ) of CD36-positive platelets increased significantly stimulated by IL-6-PMVs, with similar effect as $10 \mu \mathrm{mol} / \mathrm{L}$ ADP (Fig. 2a). CD36 deficiency in platelets prevent the effect of IL-6-PMVs while CD36-deficient platelets respond normally to ADP (Fig. 2b).

\section{TS IIA blocks platelet CD36}

To test the effect of TS IIA on platelet CD36, a receptor of PMVs, we treated resting platelets $\left(1 \times 10^{6} / \mathrm{mL}\right)$ with serial doses of TS IIA $(5-100 \mu \mathrm{g} / \mathrm{mL})$ before incubation with PE-conjugated anti-CD36 antibody. The expression of platelet CD36 did not change in response to IL-6PMVs. TS IIA could prevent the binding of PEconjugated anti-CD36 antibody to platelets in a concentration-dependent manner. TS IIA with the concentration of $20 \mu \mathrm{g} / \mathrm{mL}$ decreased the expression of CD36 significantly. With the increase of TS IIA concentration, the inhibitory action is stronger (Fig. 3a).

\section{TS IIA prevents platelet activation}

To further examine the effect of TS IIA on platelet CD36 level, we determined the expression of integrin $\alpha \operatorname{IIb} \beta 3$, a platelet activation marker. IL-6-PMVs significantly enhanced the expression of integrin $\alpha \operatorname{IIb} \beta 3$, which could be attenuated significantly by $10 \mu \mathrm{g} / \mathrm{mL}$ TS IIA. In addition, TSIIA $(5-100 \mu \mathrm{g} / \mathrm{mL})$ dose-dependently prevented the activation of platelets (Fig. 3b).

\section{A}

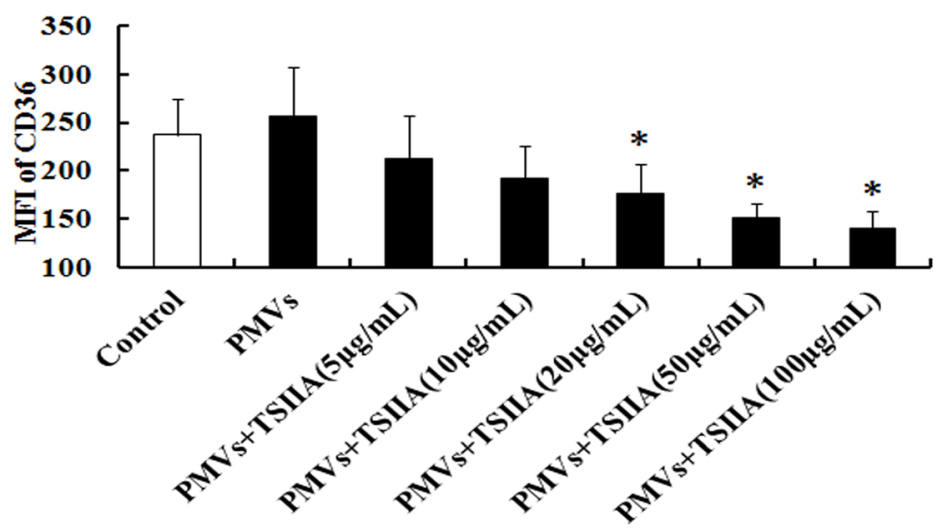

B

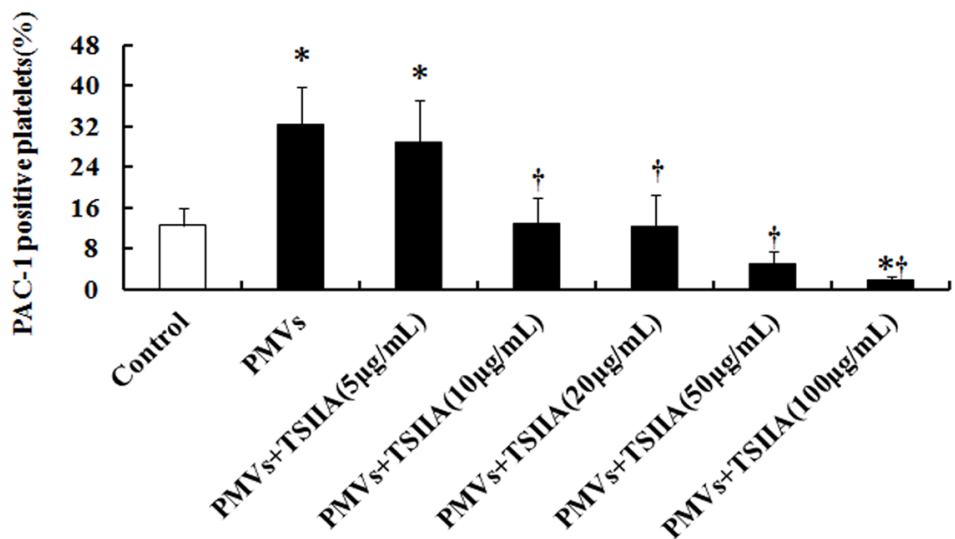

Fig. 3 Effect of TanshinonellA (TS IIA) on platelet CD36 level and platelet activation. Quantification of flow cytometry of platelets incubated with serial concentrations of TS IIA for 15 min before stimulation with $10 \mu \mathrm{g} / \mathrm{mL}$ PMVs, then CD36 MFI a and PAC-1 positivity b was measured. Data are means $\pm \mathrm{SD}(n=4)$. ${ }^{*} P<0.05$ compared with untreated control. \# $P<0.05$ compared with PMV treatment 
TSIIA downregulates the PMV-activated phosphorylation of MKK4/JNK2

Next, we tested the effect of TS IIA on the MKK4/JNK2 signal pathway by immunoblotting. Resting platelets exposed to IL-6-PMVs for $30 \mathrm{~min}$ showed a significant increase in phosphorylation of JNK2 and its upstream activator MKK4. As expected, TS IIA administration lead to downregulation of the phosphorylation of MKK4/JNK2 in platelets pretreated by IL-6-PMVs. The effect of TS IIA was comparable to that of SP600125, a pharmacological inhibitor of JNK (Fig. 4).

\section{Discussion}

Our work showed that TS IIA in vitro inhibits, in a concentration dependent manner, platelet activation induced by PMVs and downregulates CD36 and MKK4/JNK2 signaling pathway. CD36 is speculated to be attractive target of atherosclerosis (AS)-related thrombosis.

TS IIA is a diterpene quinone isolated from dried roots of salvia miltiorrhiza bunge, also known as Danshen, that has traditionally been used in Asian countries for the treatment of atherosclerotic disorders,

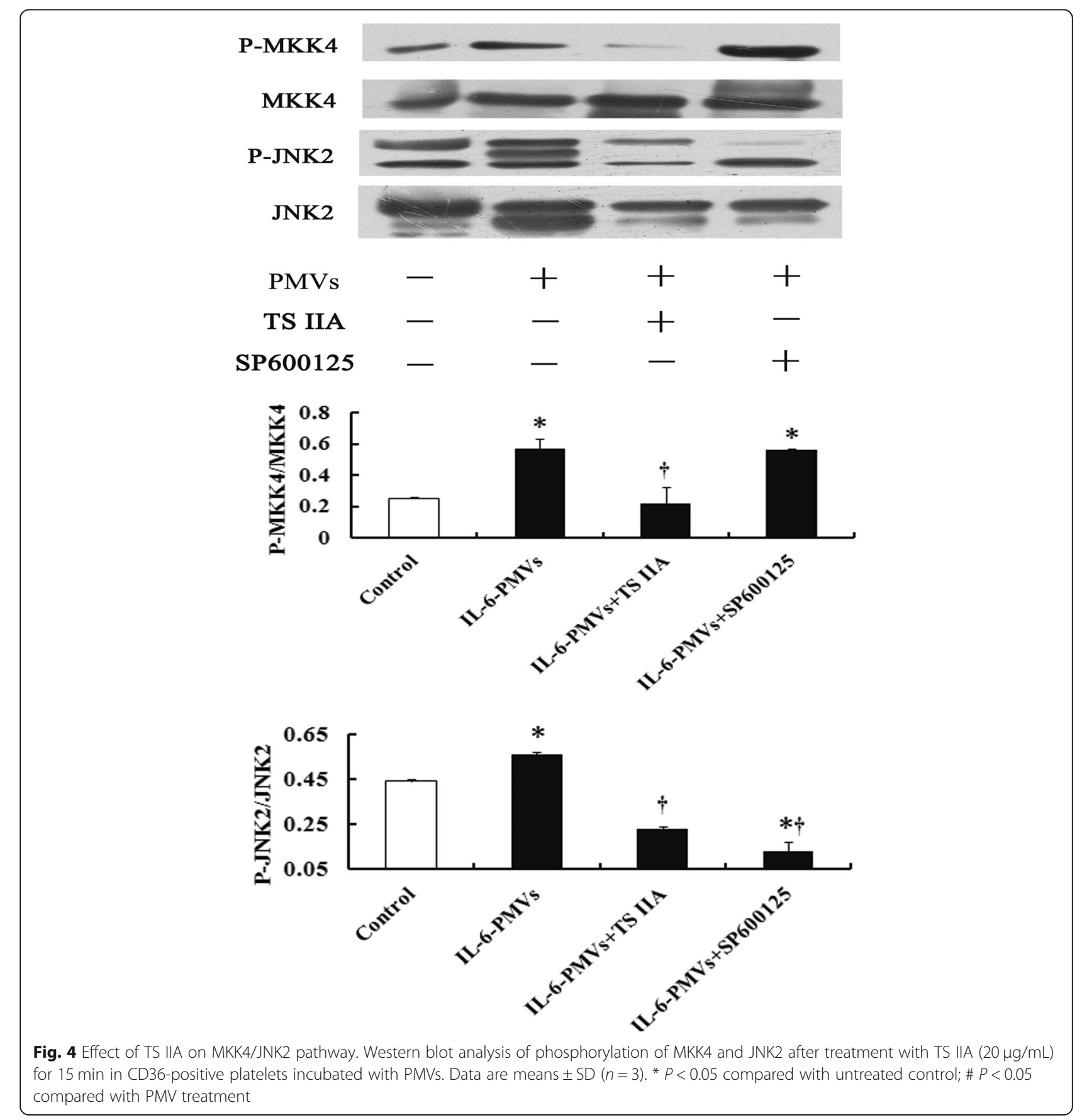


including coronary artery disease, myocardial infarction and stroke. Thromboembolic events are lethal consequences of AS. More than 150 years ago, Virchow proposed a triad of events needed for thrombus formation-abnormalities of the vessel wall, blood flow, and blood constituents [15]. An update of Virchow's triad for thrombus formation can be considered as: endothelial dysfunction; abnormal blood stasis; and abnormal platelets, coagulation, and fibrinolysis [16]. Platelets are the central part of the blood constituents, which have long been implicated in the pathogenesis of AS as major components of thrombosis, or as constituents of atheroma [3]. Although there is evidence showing that TS IIA has an anti-platelet effect, its underlying mechanism of anti-platelat effect is not very clear. Here, we investigated the effect of TS IIA on platelet activation and demonstrated that platelet CD36 may be the target of TS IIA.

CD36 is a multifunctional glycoprotein that contributes to several important pathological processes highly relevant to AS. Mice with CD36 deficiency exhibited a greater than $77 \%$ decrease in aorta lesions despite the induction of a very high atherogenic milieu [17]. Geloen et al. identified two CD36 inhibitors which were able to rescue well characterized animal models from AS [18]. In the previous study [11], we found that PMVs were ineffective for CD36-deficient platelets. The PMVs derived from oxidized Low Density Lipoprotein (oxLDL)-stimulated platelets could not enhance integrin $\alpha \operatorname{IIb} \beta 3$ expression in CD36-deficient platelets. The volunteers with CD36 deficiency have not any history of bleeding diathesis. In the present study, we found that TS IIA could decrease the expression of platelet CD36, leading to inhibition of platelet activation.

We have showed that the PMV-CD36 complex activates MKK4/JNK2 signals and contributes to platelet activation [11]. Earlier studies demonstrated that TS IIA can reduce oxidative stress and regulate apoptosis by suppressing JNK and p38 MAPK activation [19]. Nevertheless, the effect of TS IIA on platelet MAPK signal pathways is unknown. Therefore, we explored whether the signal pathway mediated by PMV-CD36 complex could be a potential target for preventing the AS-related thrombosis. Phosphorylation of JNK2 is the end-stage of the pathway; however, inhibition of the MKK/JNK pathway may induce unexpected complications because the activation of the MKK/JNK signal pathway regulates a range of biological processes and occurs inside platelets [20, 21]. The PMVCD36 complex on the surface of platelets is easier to block. Thus, we focused on targetting the PMVCD36 complex. TS IIA could block platelet CD36 expression, reduce the production of PMVs induced by IL-6 and inhibit platelet activation. In addition, TS
IIA could down-regulates the MKK4/JNK2 phosphorylation. Finally, TS IIA could inhibit the platelet activation stimulated by PMVs. Our results are consistent with several studies showing that TS IIA could inhibit platelet aggregation [22-24]. The PMV-CD36 complex may be a target for preventing the AS-related thrombosis. Of course, there are many other proteins that have influence on the MKK4/JNK2 signaling pathway. This study mainly focus on the PMV-CD36 complex, others also may need to be studied in future.

\section{Conclusion}

In conclusion, our results indicated that TS IIA could inhibit platelet activation induced by PMVs and downregulates CD36 and MKK4/JNK2 signaling pathway. This study may provide a new strategy for the treatment of AS-related thrombosis by targeting platelet CD36.

\section{Abbreviations}

ADP: Adenosine diphosphate; AS: Atherosclerosis; BSA: Bovin serum albumin; FITC: Fluorescein isothiocyanate; IL-6: Interleukin 6; IL-6-PMVs: Microvesicles derived from IL-6 stimulated platelets; JNK2: Jun N-terminal kinase 2; MKK4: Mitogen-activated protein kinase kinase 4; oxLDL: Oxidized low density lipoprotein; PE: Phycoerythrin; p-JNK: Phospho-JNK; p-

MKK4: Phospho-MKK4; PMVs: Platelet-derived microvesicles; PRP: Platelet-rich plasma; TS IIA: Tanshinone ॥A

\section{Acknowledgements}

Not applicable.

\section{Authors' contributions}

HW and LZ designed the study, performed the experiments and drafted the article. SM and NS contributed to data collection and analysis. WZ and MZ were involved in study design, data interpretation and manuscript revision. All authors edited and approved the final manuscript.

\section{Funding}

This work was supported by the research grants from the National Natural Science Foundation of China $(81471036,81873534,81702194,81600633$, $81670411,81570400,81801953,81470560)$, Key research and development program of Shandong Province (2018GSF118002, 2018GSF118017), the Natural Science Foundation of Shandong Province (ZR2017LH004, ZR2014HQ037, ZR2017BH023), the Science and Technology planning project of Yantai City (2016ZH083, 2018SFGY090). The funding bodies did not have any role in the design of the study, data collection and analysis, nor on the interpretation and dissemination of the results.

\section{Availability of data and materials}

The datasets used and/or analysed during the current study available from the corresponding author on reasonable request.

\section{Ethics approval and consent to participate}

The study was conducted in accordance with the Declaration of Helsinki, and was approved by the Human Research Ethics Committee of Shandong University. All participants provided written informed consent.

\section{Consent for publication}

Not applicable.

\section{Competing interests}

The authors declare no competing financial interests of this article.

\section{Author details}

${ }^{1}$ The Key Laboratory of Cardiovascular Remodeling and Function Research, Chinese Ministry of Education, Chinese National Health Commission and 
Chinese Academy of Medical Sciences, The State and Shandong Province Joint Key Laboratory of Translational Cardiovascular Medicine, Department of Cardiology, Qilu Hospital of Shandong University, No 107 Wenhua West Road, Ji'nan 250012, China. ²Department of Cardiology, Yantai Yuhuangding Hospital, Qingdao Medical College, Qingdao University, Yantai, China.

Received: 29 June 2019 Accepted: 29 November 2019

Published online: 14 February 2020

\section{References}

1. Wang X, Morris-Natschke SL, Lee KH. New developments in the chemistry and biology of the bioactive constituents of Danshen. Med Res Rev. 2007; 27:133-48.

2. Gao S, Liu Z, Li H, Little PJ, Liu P, Xu S. Cardiovascular actions and therapeutic potential of tanshinone IIA. Atherosclerosis. 2012;220:3-10

3. Chung I, Lip GY. Virchow's triad revisited: blood constituents. Pathophysiol Haemost Thromb. 2003;33:449-54.

4. Shantsila E, Kamphuisen PW, Lip GY. Circulating microparticles in cardiovascular disease: implications for atherogenesis and atherothrombosis. J Thromb Haemost. 2010;8:2358-68.

5. Cocucci E, Racchetti G, Meldolesi J. Shedding microvesicles: artefacts no more. Trends Cell Biol. 2009;19:43-51.

6. Date $\mathrm{K}$, Ettelaie C, Maraveyas A. Tissue factor-bearing microparticles and inflammation: a potential mechanism for the development of venous thromboembolism in cancer. J Thromb Haemost. 2017;12:2289-99.

7. Fan G, Jiang X, Wu X, Fordjour PA, Miao L, Zhang H, et al. Anti-inflammatory activity of tanshinone IIA in LPS-stimulated RAW264.7 macrophages via mirnas and TLR4-NF-KB pathway. Inflammation. 2016;39:375-84.

8. Cao EH, Liu XQ, Wang JJ, Xu NF. Effect of natural antioxidant tanshinone IIA on DNA damage by lipid peroxidation in liver cells. Free Radic Biol Med. 1996;20:801-6

9. Fei YX, Wang SQ, Yang LJ, Qiu YY, Li YZ, Liu WY, et al. Salvia miltiorrhiza Bunge (Danshen) extract attenuates permanent cerebral ischemia through inhibiting platelet activation in rats. J Ethnopharmacol. 2017;207:57-66.

10. Tang FT, Cao Y, Wang TQ, Wang LJ, Guo J, Zhou XS, et al. Tanshinone IIA attenuates atherosclerosis in $\mathrm{ApoE}(-/-)$ mice through down-regulation of scavenger receptor expression. Eur J Pharmacol. 2011;650:275-84.

11. Wang $H$, Wang $Z H$, Kong J, Yang MY, Jiang GH, Wang XP, et al. Oxidized low-density lipoprotein-dependent platelet-derived microvesicles trigger procoagulant effects and amplify oxidative stress. Mol Med. 2012;18:159-66.

12. Ghosh A, Li W, Febbraio M, Espinola RG, McCrae KR, Cockrell E, et al. Platelet CD36 mediates interactions with endothelial cell-derived microparticles and contributes to thrombosis in mice. J Clin Invest. 2008;118:1934-43.

13. Heijnen HF, Schiel AE, Fijnheer R, Geuze HJ, Sixma JJ. Activated platelets release two types of membrane vesicles: microvesicles by surface shedding and exosomes derived from exocytosis of multivesicular bodies and alphagranules. Blood. 1999;94:3791-9.

14. Kashiwagi H, Tomiyama Y, Nozaki S, Kiyoi T, Tadokoro S, Matsumoto K Analyses of genetic abnormalities in type I CD36 deficiency in Japan: identification and cell biological characterization of two novel mutations that cause CD36 deficiency in man. Hum Genet. 2001;108:459-66.

15. Brotman DJ, Deitcher SR, Lip GY, Matzdorff AC. Virchow's triad revisited. South Med J. 2004;97:213-4.

16. Watson T, Shantsila E, Lip GY. Mechanisms of thrombogenesis in atrial fibrillation: Virchow's triad revisited. Lancet. 2009;373:155-66.

17. Febbraio M, Podrez EA, Smith JD, Hajjar DP, Hazen SL, Hoff HF, et al, Targeted disruption of the class B scavenger receptor CD36 protects against atherosclerotic lesion development in mice. J Clin Invest. 2000;105:1049-56.

18. Geloen A, Helin L, Geeraert B, Malaud E, Holvoet P, Marguerie G. CD36 inhibitors reduce postprandial hypertriglyceridemia and protect against diabetic dyslipidemia and atherosclerosis. PLoS One. 2012;7:e37633.

19. Li H, Han W, Wang H, Ding F, Xiao L, Shi R, et al. Tanshinone IIA inhibits glutamate-induced oxidative toxicity through prevention of mitochondrial dysfunction and suppression of MAPK activation in SH-SY5Y human neuroblastoma cells. Oxidative Med Cell Longev. 2017;2017:4517486.

20. Whitmarsh AJ, Davis RJ. Transcription factor AP-1 regulation by mitogenactivated protein kinase signal transduction pathways. J Mol Med. 1996;74: 589-607.

21. Davis RJ. Signal transduction by the JNK group of MAP kinases. Cell. 2000; 103:239-52.
22. Lin HC, Ding HY, Chang WL. Two new fatty diterpenoids from Salvia miltiorrhiza. J Nat Prod. 2001;64:648-50.

23. Lee AR, Wu WL, Chang WL, Lin HC, King ML. Isolation and bioactivity of new tanshinones. J Nat Prod. 1987:50:157-60.

24. Wang $\mathrm{N}$, Luo HW, Niwa M, Ji J. A new platelet aggregation inhibitor from Salvia miltiorrhiza. Planta Med. 1989:55:390-1.

\section{Publisher's Note}

Springer Nature remains neutral with regard to jurisdictional claims in published maps and institutional affiliations.
Ready to submit your research? Choose BMC and benefit from:

- fast, convenient online submission

- thorough peer review by experienced researchers in your field

- rapid publication on acceptance

- support for research data, including large and complex data types

- gold Open Access which fosters wider collaboration and increased citations

- maximum visibility for your research: over $100 \mathrm{M}$ website views per year

At BMC, research is always in progress.

Learn more biomedcentral.com/submissions 